\title{
The End of Monogamy? An Exploration of Non-monogamous Relationship Dynamics
}

\author{
Carly Bruce ${ }^{1}$
}

\begin{abstract}
Although monogamy is the dominant relationship style in the Western world, there are alternative options for non-monogamous relationship dynamics. This paper works to explore how the roots of how monogamy became the dominant structure, the hidden drawbacks of monogamy, how those who assert monogamy to be compulsory hold a stigma against those who partake in other dynamics, and how this stigma can work to oppress. It also explores what those dynamics are, who participates in them, and a myriad of benefits that non-monogamous relationships can have. Overall coming to the conclusion that everyone should be able to make an informed decision, free of societal judgment, to partake in whatever dynamic they may choose.
\end{abstract}

\section{Introduction}

When it comes to deciding on our romantic relationship paths, many of us will wind up searching out a single romantic partner that we can commit to exclusively; this paper will explore why that seems to be the default, some other relationship dynamics and the stigma against these alternatives. Monogamy is the relationship dynamic that is most widely accepted in the Western world, and is one that relies on an ideally lifelong commitment (often marital) to a single other person (Rothschild, 2018). The dominant concept that monogamy is the best, most moral, healthy, normal, and ideal relationship structure is called mononormativity or can also be referred to as compulsory monogamy (Rothschild, 2018).

It is worth noting that other relationship structures and dynamics are legitimate and can be just as healthy, even though they are highly stigmatized in Western society. Consensual non-monogamy $(C N M)$ is an umbrella term for many types of relationships that do not limit themselves to only having one partner, with variation surrounding agreed upon limits when it comes to sexual and/or emotional relationships with other people (Sheff, 2020). One branch of CNM that this paper will focus on exploring is polyamory, "in which people are allowed to engage in more than one sexual and/or romantic and or/intimate relationship at the same time,

\footnotetext{
${ }^{1}$ MacEwan University, Edmonton, Alberta
} 
with the informed consent of all parties" (Rothschild, 2018, p. 42). The inclusion of informed consent from all parties is an important distinction between polyamory and infidelity and cheating in a monogamous relationship; which Sheff (2020) notes is inherently nonconsensual and doesn't involve discussion or negotiation from all people involved.

\section{Literature Review}

There has been plenty of research done exploring monogamy, the benefits of monogamy (York, 2020), how it can become an oppressive experience (Rothschild, 2018 \& Sheff, 2020), as well as exploring different alternatives to monogamy and their possible benefits (Moors, Matsick, and Schechinger, 2017). Stigma surrounding non-monogamy and the effects of this stigma were studied extensively in works done by Mogilski, et. al., (2020) as well as Cassidy et al. (2018), and Rotschild (2018). They have done these studies based on attitudes formed my both people who engage solely in monogamy, as well as those who desire to, or have engaged in, polyamorous and other CNM relationship dynamics (Moors, Gesselman, and Garcia, 2021). Throughout reviewing all of this literature and past research that has been completed, this paper seeks to measure the positive and negative aspects of both monogamy and $\mathrm{CNM}$ relationship styles while working to remove some of the stigma and misinformation surrounding non-monogamous relationship dynamics so readers can make more informed decisions when it comes to their own relationships, as well as what judgments they pass on those who make decisions that differ from their own.

\section{What is Monogamy?}

Monogamy is the most represented and accepted form of romantic relationship in the Western world (Rothschild, 2018). We see it in exemplified and upheld as the ideal in most, if not all, of our movies, television shows, stories, music and other forms of popular media. The fairy tale girls are told from a young age is one of a monogamous love; that one day they will find their one true love, get married, start a family, and eventually live happily ever after. The popular public discourse surrounding monogamy is that it is the default relationship, making any other relationship seem unimaginable and, to some, immoral (Rothschild, 2018). This initial judgment of it being unimaginable or immoral is illustrated in Sheff's (2020) exploration of three common reactions to learning about polyamory and the concept that there is alternatives to monogamy to which fall under categories of: excitement at learning there is a different option that may suit them better, indifference, or moral terror at the very concept of straying from monogamy.

Sheff (2020) discusses two separate categories of monogamy. The first is traditional monogamy, in which a person refrains from engaging in intercourse before marriage and then only engages in sexual acts with the person they are married to (p.885). This traditional type of monogamy is largely rooted in Christianity and the importance of using sex primarily for procreation (Sheff, 2020). Traditional monogamy was also a lot more prevalent when life spans were shorter and the lack of travel and resources available meant that you met far fewer people (Rothschild, 2018). The second category of monogamy that Sheff (2020) investigates is what 
is referred to as serial monogamy "in which each person has one partner at a time, breaks up, and re-partners with one other person, repeatedly until permanent partnership is established or death" (p. 886). This is the image of monogamy we are generally presented with as the correct path to take when we observe public discourse and popular media.

\section{Oppression and Infidelity within Monogamy}

While discussing how society upholds this type of serial monogamy as the norm and the proper way to conduct romantic relationships, we must also acknowledge that it can create a gendered power imbalance that can easily become oppressive, especially when discussing heterosexual relationships (Rothschild, 2018). This oppression, of women specifically, can be seen partially through how the legal system has dealt with various issues between a married couple; issues including how women are often treated more harshly in a divorce proceeding if they were the ones who committed adultery (compared to if the man committed the same adulterous acts) (Rothschild, 2020). Another example of this gendered oppression is how marital rape didn't used to be considered rape in many countries, as the wife was seen as property of the man and providing sex whenever he wanted was just part of the job of being a wife (Rothschild, 2020). Even outside of Western modern society, this gendered double standard has been prevalent throughout history, many allowing men numerous acceptable extramarital romantic and/or sexual relationships where they had "concubines, whores, saltwives, courtesans and mistresses" (Rothschild, 2018, p. 31) while demanding monogamy from women who can face severe punishment and ridicule if they were to stray. Rothschild (2018) also discusses the dichotomy of condoning and encouraging men to have multiple sexual partners, while women with the same number of partners are condemned and labeled as 'sluts'.

Infidelity within marriages is a significant issue, with some conservative estimates stating that at least $25 \%$ of married people engage in infidelity at some point throughout the duration of their marriage (Sheff, 2020). Sheff (2020) works to discuss how having a society that is structured around compulsory monogamy while also having infidelity be a fairly normalized and popular practice, presents us with an imbalanced and largely oppressive framework for relationships. Cheating and acts of infidelity create an inherent power imbalance, as the person who believes they are in a monogamous relationship when they are being lied to is instantly disadvantaged as they are less aware of the reality of the relationship (Sheff, 2020). This type of imbalance leads to all the power being placed in the hands of the one cheating, and can humiliate the faithful partner with or without their knowledge (Sheff, 2020)

When fielding discussion surrounding infidelity in monogamy, it is essential to consider how some people can use the CNM title on dating apps or other interactions outside of their relationship to more easily engage in cheating on their partners in a relationship that the other partner believes to be monogamous, as well as justifying their behaviour to themselves by stating they are inherently non-monogamous (De Graeve, 2018). The practice of men using $\mathrm{CNM}$ to engage in extramarital relationships with women without their partners knowledge was explored by De Graeve (2018). Many of the men in this study used the label of CNM to tell themselves that they were still good men that were protecting their monogamous partners by keeping it a secret while feeding their traditionally masculine sexual appetites; most of them committing to finding outside sexual relationships with no emotional attachment (De Graeve, 2018). Secretly cheating, being so popular, actually works 
to reinforce compulsory monogamy, where the open dialogue of real CNM relationships shakes the foundations of a society structured around monogamy; so, the fact that people are using CNM to hide infidelity only works to further stigmatize CNM relationships (Sheff, 2020). If infidelity and violence were reacted to equally by society and engaged in an equal amount by male and female partners in heterosexual and monogamous relationships, maybe a case could be made that compulsory monogamy should be the standard that everyone strives for. However, with the imbalanced power dynamic as it stands currently, forcing people to engage in monogamy may not be as advantageous as we are led to believe.

\section{Justifications for Monogamy}

While monogamy, and more specifically, compulsory monogamy, may have some oppressive and gendered aspects while being rooted in times when people died at earlier ages, couldn't travel as far, and more of society was based around Christian values, monogamy may not always be the wrong answer. In a response to recent attacks on monogamy as being corrupt and inherently oppressive, York (2020) works to defend some common justifications of monogamy and states it is also morally permissible. The three main justifications presented by York (2020) are the practicality of having only one partner, the special feeling a person can get from being the exclusive partner of the person they love, and avoiding confronting jealous thoughts that are perceived by monogamous people to have the potential to be more apparent and dominating in a CNM relationship.

Practicality can come into play when it comes to the formal aspects of becoming legally married and parenting in relationships. Although an argument could be made that having more streams of income in a home and more adults to help raise a child could be advantageous, it is not the accepted norm of the majority at this time. York (2020) discusses how only having one partner is more practical, as there are fewer people to split your time, attention, and any other resources you may be providing. As a result, the one partner situation may be less emotionally taxing on each individual.

The special feeling between a couple is what we are shown in Disney movies where the princess finds her prince, or in the popular media when someone finds "the one". The characters feel special and whole and like they are living in a world of their own. One also feels special when they feel like they have exclusive access to a person or experience or when they feel like a person considers them better or more suitable than anyone else, which is the assumption made when participating in a monogamous relationship (York, 2020). Although jealousy can happen to anyone in any interpersonal context, a case could be made that only having one partner and being in a completely monogamous relationship could make a partner feel more justified in being jealous, as jealousy in monogamous relationships is often seen as "evidence of true love" (Sheff, 2020, p. 884). Specific examples of these special circumstances that many people think are inherent in a CNM relationship, could include feeling like you are not enough for your partner, being scared they will like the other person more than they like you and you'll get left behind, or being emotionally or sexually neglected when they are involved with another person (York, 2020). 


\section{What is Polyamory and Who is Polyamorous?}

Polyamory is one form of a CNM relationship dynamic that is said to have started during the free love movement in the 1970s and became highly popularized in the 1990s (Sheff, 2020). Polyamorous relationships can have plenty of individual variation in structure, but all generally depart from the monogamous idea that one partner should meet all of an individual's romantic, intimate, and sexual needs (Rothschild, 2018). A 2021 study by Moors, Gesselman, and Garcia focused on desire, familiarity, and engagement with polyamory in the United States and found that one in six participants had some level of desire to engage in polyamory, one in nine had already engaged in polyamory, one in fifteen knew someone in their life who had taken part, and of those who had no desire to engage in polyamory one in seven respected people who engaged in polyamory. What distinguishes polyamory from other CNM dynamics (like swinging or open relationships which will be discussed later), is that the participants in the relationship are encouraged to seek out romantic love as well as sexual activity with multiple partners, rather than purely physical/sexual encounters (Moors, Gesselman, and Garcia, 2021). This is not to say that all polyamorous arrangements allow the original two partners to engage in romantic and/or sexual relationships with everyone and anyone; some dynamics have a finite limit for how many people are to be involved as well as different guidelines on whether they date independently or have any additional partners date both the original partners as in a triad dynamic where all three individuals involved are in a collective relationship (Moors, Gesselman, and Garcia, 2021). When examining demographic information, Moors, Gesselman, and Garcia (2021) found that "sexual minorities, men, and younger adults reported greater desire to engage in polyamory [while] men and people with lower education backgrounds were more likely to have previously engaged in polyamory" ( $p$. 1).

\section{Stigma and Misunderstandings about Polyamory and CNM}

As has been alluded to through some of the discussion surrounding compulsory monogamy, there are many misunderstandings about polyamory and CNM relationships that can attribute to stigma and resulting discrimination against participants of any form of nonmonogamous relationship. As mentioned, De Graeve (2018) explored men using the label of CNM as a cover to engage in infidelity behind their partner's backs. This feeds into the general myth that CNM and polyamory is just some sort of cheating in disguise. Rothschild (2018) assures that one of the core components of a legitimate CNM relationship is that all participants are consenting to the addition of new relationships of varying levels of intimacy and sex. This disproves the 'cheating in disguise' narrative that many label CNM relationships with.

Another misconception commonly held by people who only engage in monogamy is that there must be more jealousy in CNM relationships due to the involvement of additional people (Mogilski, et al., 2020). It seems that someone is always excluded and that there must be a constant state of competition between partners (Mogilski, et al., 2020). This train of thought could be derived from zero-sum thinking about the abundance or scarcity of love which essentially states that "one person's love gained is another's love lost" (p. 25) which therefore must mean that seeking additional partners means a person loves the first partner less (Burleigh, et. al., 2017). Zero-sum thinking is also part of why the practicality defence 
made earlier seems so palatable to people who reinforce compulsory monogamy; the belief that a person only has a finite amount of love, attention, and emotional fuel must mean that they should be investing all of those resources into one person so they don't spread themselves too thin and neglect their primary partner (Burleigh, et al., 2017; York, 2020).

The Christian foundation of compulsory monogamy also contributes to the idea that any relationship dynamic outside a strict monogamous arrangement is morally and ethically corrupt (Rothschild, 2018). This is a huge contributor to the stigma against polyamory and other CNM relationships (Rothschild, 2018). One misconception that often pairs with the immorality of CNM relationship dynamics, is that having multiple sexual partners is a greater risk to sexual health (Moors, Matsick, and Schechinger, 2017). At first glance, this may seem like a logical assumption to make, but the fact is that $91 \%$ of CNM people in a study had explicit boundaries when it came to sexual health and used condoms and barriers more consistently than monogamous couples (who usually eventually stop using protection at some point into their relationship) (Moors, Matsick, and Schechinger, 2017). When you consider this in conjunction with the statistics on how often monogamous couples engage in infidelity, a case could be made for CNM relationships being better for sexual health, as these boundaries and practices are discussed and used more frequently (Moors, Matsick, and Schechinger, 2017).

One of the most common misconceptions about polyamory and other CNM dynamics that keep people in patterns of monogamy is that jealousy must be so much more prevalent in a relationship where you are sharing your partner with other people. Moors, Gesselman, and Garcia (2021) found this to be largely incorrect and note that jealousy is actually one of the leading reasons for divorce in monogamous couples in the United States. They also found that people in CNM dynamics are generally better at managing any jealousy they may experience, often describing it in fairly mild terms (Moors, Gesselman, and Garcia, 2021). Taking the act of engaging in multiple relationships out of the shadows and creating open discussion surrounding boundaries and any negative reactions that may bubble up is conducive to a more balanced and trusting relationship and removes the imbalance of one person better knowing the reality of the relationship (Sheff, 2020). There is less need to worry about whether your partner is lying to you or hiding something, when there is no reason to hide anything within the boundaries of the agreed upon dynamic. Although this is not to say that some people still abuse these boundaries or that there is a complete freedom from any jealousy or codependency (Rothschild, 2018).

\section{Negative Outcomes of Stigma Against CNM}

Labeling a group as wrong or corrupt can be very alienating no matter what that group is and can lead to harmful discriminatory practices; those engaging in polyamory and other forms of CNM relationships are not immune to this effect. Possible rejection from family, friends, and society in general can be incredibly harmful to an individual's well-being and this condemnation leads many polyamorous and CNM individuals to hide their relationship dynamic (Mogilski, et al., 2021). This sheltering of a person's dynamic is similar to how someone who identifies as part of the LGBTQIA+ communities may stay 'in the closet' because they fear being ostracized (Mogilski, et al., 2021). This fear of disapproval and stigmatization can also lead to being more secretive about any partners other than the 'primary' or original partner (Mogilski, et al., 2021) and this could conceivably lead to the 
other partners feeling less important or valued and could push people who want to engage in polyamory into lives more consistent with compulsory monogamy.

Another important consequence of polyamory and CNM being highly stigmatized in Western society is how it can affect the frequency at which these individuals seek out medical and psychological assistance and how they are treated in these environments. Mogilski, et al. (2021) found that "fear of judgment can cause anxiety that prevents those who practice CNM from seeking sexual health services (e.g., STD testing)" (p. 8). There is already stigma surrounding testing for sexually transmitted diseases and infections, so adding the layer of stigma against the relationship dynamic a person may be consensually engaging in is incredibly harmful and dangerous. Although psychotherapists and sexologists are supposed to be free of bias and knowledgeable about many forms of relationships and practices, the field of counselling is not free of bias based on the dominant discourse of mononormativity (Cassidy and Wong, 2018). Therapists with little or no experience or knowledge about CNM dynamics may have difficulty distinguishing between extramarital affairs (infidelity) and co-marital relationships (agreed upon by all members) (Cassidy and Wong, 2018). They may also have offices too small and appointment times too short to accommodate a triad or quad polyamorous relationship (Cassidy \& Wong, 2018). It was also found that clients who are in CNM relationships stated (unsurprisingly) that the therapists that were most helpful were those that were affirmative, rather than condemning, of their relationship dynamic (Mogilski, et al., 2020).

\section{Benefits of Polyamory and CNM Relationship Dynamics}

After examining the dominating discourse surrounding compulsory monogamy and some of the stigma and misconceptions present in regards to polyamory and other CNM relationship dynamics one may be left wondering why anyone would willingly go into such a confusing and stigmatized relationship. If one is able to look beyond the outside judgment and possible drawbacks, the myriad of benefits would become apparent. Benefits that include leveling the gendered power imbalance, negating toxic ideas of equating jealousy with love, encouraging boundaries and self-sufficiency, and opening oneself up to greater fulfillment and personal growth (Rothschild, 2018 and Moors; Matsick and Schechinger, 2012).

Rothschild (2018) argues that the driving concept behind CNM inherently and immediately subverts the gender imbalance in heterosexual monogamy, that treats women more harshly for going beyond a single partner and by allowing men and women the equal opportunity to find additional emotional and/or sexual partners. It also works to fight against the equating of jealousy and possessiveness with true love, by acknowledging that love is not finite and does not have to be limited to a single partner while simultaneously encouraging open discussion around boundaries and any jealous feelings that may participants may experience (Rothschild, 2018).

In popular media, as well as in real life, we have all seen instances of the 'two become one' rhetoric in monogamous relationships where the two individuals in a relationship tend to become more and more similar as they withdraw from larger social networks (with some exception to spending time with other monogamous couples). Moors, Matsick, and Schechinger (2017) found that individuals in CNM relationships experience a higher rate of variety in nonsexual and sexual activity (which varies in different dynamic styles) than monogamous couples would, as well as experiencing less of a dyadic withdrawal 
from their social network. This becomes especially apparent when one partner has hobbies and interests that differ from any of the other partners and gives the opportunity for one to expand their horizons and participate in a wider variety of nonsexual activities (Moors, Matsick, and Shechinger. 2017).

The concept of needs being fulfilled by more than just a single monogamous partner is also highly beneficial to participants in CNM relationships, as they can get a better variety of needs met when interacting with multiple people (Moors, Matsick, and Schechinger, 2017), as well as increased focus on self-sufficiency (Rothschild, 2018). When discussing Maslow's hierarchy of needs (which includes physiological needs, safety, belonging, and selfactualization) as well as needs determined by Self- Determination theorists (including aspects pertaining to categories such as autonomy and relatedness), it seems like a lot to place on the shoulders of a single person you love and are also trying to provide these things for (Moors, Matsick, and Schechinger, 2017). It was also specified that people who engage in polyamory are not doing it because a primary partner is not sufficiently meeting their needs, but rather they found that having multiple partners met a greater variety of needs (Moors, Matsick, and Schechinger, 2017). They seek out polyamory not out of a state of scarcity, but rather they are seeking out even greater fulfillment, which directly goes against the zero-sum thinking explored by Burleigh, et al. (2017). Polyamory, with its allowance for romantic/emotional as well as sexual relations with others, helps to spread the fulfillment of this list of needs across multiple people while also holding oneself accountable to work on fulfilling themselves and others (Rothschild, 2018). Not relying on any single person to provide all of this or limiting oneself to a monogamous experience can result in simultaneous feelings of both freedom and security which, in turn, reinforces a higher level of feelings of autonomy over ones life which leads to higher states of well-being (Moors, Matsick, and Schechinger, 2017).

While this paper has been focused on going beyond just sexual aspects of CNM relationships, these dynamics do also encourage variety in sexual partners and sexual experiences. This sexual freedom CNM allows encourages exploration of an individual's sexuality and offers non-heterosexual individuals an opportunity to take part in sexual relationships with people who have gender identities that differ from their primary partner without any guilt or shame (Moors, Matsick, and Schechinger, 2017). This freedom to experience both emotional and sexual connections with people of multiple gender identities can be paramount for individuals who identify as bisexual or pansexual (Moors, Matsick, and Schechinger, 2017). It is not to say that those who identify with these sexual orientations cannot also be happy in monogamous relationships if they choose to be. Even when the outside relationships that are allowed are purely sexual, as is the case with swingers (who will be discussed in the next section), it was found that over $75 \%$ of swingers in a study found their lives to be exciting overall, in comparison to less than $50 \%$ of those included in a national survey done in the United States on adult life satisfaction published in 2010; it was assumed that most people in this survey were monogamous (Moors, Matsick, and Schechinger, 2017).

\section{Forms of CNM Other Than Polyamory}

While this paper has sought to explore the world of CNM beyond just the sexually adventurous aspects that are often focused on when prescribing CNM as crazy or immoral in a society dominated by compulsory monogamy, there are perfectly valid CNM dynamics that are focused on additional sexual experiences outside the primary relationship. One example 
of a sex-for-fun focused form of a CNM relationship would be what is referred to as swinging, which often involves multiple couples temporarily swapping partners for the sole purpose of sexual enjoyment, or having sex parties with a larger group of consenting participants (Sheff, 2020). This is often what people who don't engage in CNM, or those who are condemning it, are imagining any and every CNM dynamic looks like which makes it an easy target to be painted in some sexually adventurous and wild imagery. The over-representation of swinging as the only form of $\mathrm{CNM}$ also tends to trigger people who have experienced cheating or jealousy in past relationships and keeps them from considering CNM as a possibility for their own relationships (Sheff, 2020). While swinging and sex-focused branches of CNM are valid and have set boundaries with all parties consenting, the focus on these types of CNM as the only ones represented in popular media and public discourse can do a disservice to those who practice dynamics such as polyamory.

There is also a broader style of CNM dynamic that generally has less strict or specific, but still diverse, sets of boundaries. This is called an open relationship (Sheff, 2020). An open relationship could mean anything from being "non-exclusively involved with only one other person" (Mogilski, et al., 2020, p. 3), to having specific guidelines less restrictive than polyamory, but less targeted than a swinging couple. Sheff (2020) describes the title of an open relationship as one that "gives little information about the specifics of the structure beyond the fact that the participants have agreed on non-monogamy" (p. 882). While this term can be used as a broad umbrella term for many types of CNM relationships and some may choose to use it, other people in CNM dynamics may choose to use other labels. It is important to respect those choices.

\section{Conclusion}

In Western society, there is a dominating perception that monogamy should be compulsory and that any relationship that goes against monogamous ideals is immoral, depraved or shady in some way (Rothschild, 2018). Once we move past the narrow-minded and oppressive dialogue that surrounds the discourse condemning CNM relationships, it makes room to truly examine the multitude of benefits it can have for the individual, as well as for the relationship as a whole (Moors, Matsick, and Schechinger, 2017).

After examining some of the lesser-known benefits of CNM style relationships and some of the hidden drawbacks of the foundation of monogamy, it is important that we remove ourselves from any implicit biases we may have and open our minds to the possibility that CNM relationships may be the best option for some people. As is consistent with popular discourse and assumption, there are also possible drawbacks to CNM dynamics for some people and extensive benefits to monogamy, which also supports that monogamy may be the best relationship dynamic for certain people. It is this paper's position that neither of these dynamics is inherently incorrect, oppressive or immoral, and that both statements can be simultaneously correct for different people. What is most important is that we don't try to force CNM people into shame or into monogamy and that we do not try to force people who are comfortable in monogamy into CNM dynamics, but rather that we allow everyone accurate information so that they can make the decision for themselves and become more tolerant of those who make different decisions. 


\section{References}

Burleigh, T. J., Rubel, A. N., \& Meegan, D. V. (2017). Wanting 'the whole loaf': zero-sum thinking about love is associated with prejudice against consensual non-monogamists. Psychology \& Sexuality, 8(1/2), 24-40. https://doi.org/10.1080/19419899.2016.1269020

Cassidy, T., \& Wong, G. (2018). Consensually nonmonogamous clients and the impact of mononormativity in therapy. Canadian Journal of Counselling and Psychotherapy, 52(2), $119-139$.

De Graeve, K. (2019). “No expectations": Straight men's sexual and moral identity-making in non-monogamous dating. Sexualities, 22(5-6), 844-859.

https://doi.org/10.1177/1363460718779946

Mogilski, J., Mitchell, V., Reeve, S., Donaldson, S., Nicolas, S., \& Welling, L. (2020). Life History and Multi-Partner Mating: A Novel Explanation for Moral Stigma Against Consensual Non-monogamy. Frontiers in Psychology, 10.

https://doi.org/10.3389/fpsyg.2019.03033

Moors, A., Gesselman, A. \& Garcia, J. (2021). Desire, Familiarity, and Engagement in Polyamory: Results From a National Sample of Single Adults in the United States. Frontiers in Psychology, 12. https://doi.org/10.3389/fpsyg.2021.619640

Moors, A. C., Matsick, J. L., \& Schechinger, H. A. (2017). Unique and Shared Relationship Benefits of Consensually Non-Monogamous and Monogamous Relationships A Review and Insights for Moving Forward. European Psychologist, 22(1), 55-71.

https://doi.org/10.1027/1016-9040/a000278

Rothschild, L. (2018). Compulsory Monogamy and Polyamorous Existence. Graduate Journal of Social Science, 14(1), 28-56.

Sheff, E. (2020). Polyamory Is Deviant - But Not for the Reasons You May Think. Deviant Behavior. https://doi.org/10.1080/01639625.2020.1737353

York, K. (2020). Why Monogamy Is Morally Permissible: A Defense of Some Common Justifications for Monogamy. Journal of Value Inquiry, 54(4), 539-552. 